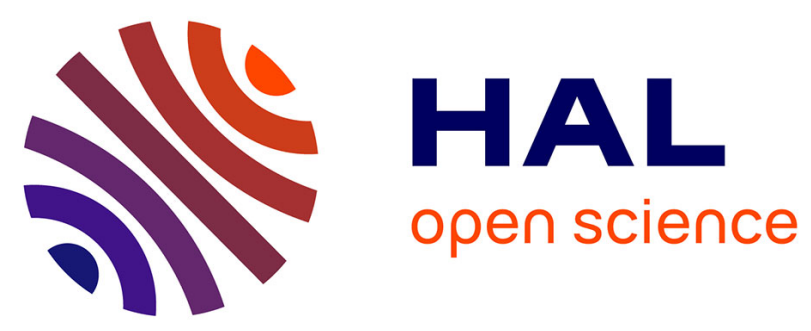

\title{
Left ventricle function assessment using gated first-pass 18F-FDG PET: Validation against equilibrium radionuclide angiography
}

Fayçal Ben Bouallègue, Laurent Maïmoun, Florentin Kucharczak, Pierre Le Fur, Fabien Vauchot, Boramy Hay, Eric Rondet, Denis Mariano-Goulart

\section{To cite this version:}

Fayçal Ben Bouallègue, Laurent Maïmoun, Florentin Kucharczak, Pierre Le Fur, Fabien Vauchot, et al.. Left ventricle function assessment using gated first-pass 18F-FDG PET: Validation against equilibrium radionuclide angiography. Journal of Nuclear Cardiology, 2021, 28 (2), pp.594-603. 10.1007/s12350-019-01731-x . hal-02119749

\section{HAL Id: hal-02119749 \\ https://hal.science/hal-02119749}

Submitted on 9 Dec 2021

HAL is a multi-disciplinary open access archive for the deposit and dissemination of scientific research documents, whether they are published or not. The documents may come from teaching and research institutions in France or abroad, or from public or private research centers.
L'archive ouverte pluridisciplinaire HAL, est destinée au dépôt et à la diffusion de documents scientifiques de niveau recherche, publiés ou non, émanant des établissements d'enseignement et de recherche français ou étrangers, des laboratoires publics ou privés. 


\title{
Left ventricle function assessment using gated first-pass ${ }^{18} \mathrm{~F}$-FDG PET: Validation against equilibrium radionuclide angiography
}

\author{
Fayçal Ben Bouallègue, MD, PhD, ${ }^{a, b}$ Laurent Maïmoun, PhD, ${ }^{a, b}$ \\ Florentin Kucharczak, PhD, ${ }^{c}$ Pierre Le Fur, D Pharm, ${ }^{a}$ \\ Fabien Vauchot, MD, PhD, ${ }^{\text {B }}$ Boramy Hay, MD, ${ }^{\mathrm{a}}$ Eric Rondet, PhD, ${ }^{\mathrm{d}}$ \\ and Denis Mariano-Goulart, MD, PhD ${ }^{\mathrm{a}, \mathrm{b}}$ \\ a Nuclear Medicine Department, Lapeyronie University Hospital, Montpellier Cedex 5, France \\ b PhyMedExp, INSERM, CNRS, Montpellier University, Montpellier, France \\ c LIRMM, Montpellier University, CNRS, Montpellier, France \\ d UMR QualiSud, Montpellier University, Montpellier, France
}

Received Feb 13, 2019; accepted Apr 12, 2019

doi: 10.1007/s12350-019-01731-x

Purpose. We appraised the feasibility of left ventricle (LV) function assessment using gated first-pass ${ }^{18}$ F-FDG PET, and assessed the concordance of the produced measurements with equilibrium radionuclide angiography (ERNA).

Materials and Methods. Twenty-four oncologic patients benefited from ${ }^{99} \mathrm{~m}$ Tc-labeled redblood-cell ERNA, in planar mode (all patients) and using SPECT (22 patients). All patients underwent gated first-pass ${ }^{18}$ F-FDG cardiac PET. Gated dynamic PET images were reconstructed over 1 minute during tracer first-pass inside the $L V$ and post-processed using in-house software (TomPool). After re-orientation into cardiac canonical axes and adjustment of the valves plane using a phase image, pseudo-planar PET images obtained by re-projection were automatically segmented using thresholded region growing and gradient-based delineation to produce an LV ejection fraction (EF) estimate. PET images were also post-processed in fullytomographic mode to produce LV end diastole volume (EDV), end systole volume (ESV), and EF estimates. Concordance was assessed using Lin's concordance (cce) and Bland-Altman analysis. Reproducibility was assessed using the coefficient of variation $(\mathrm{CoV})$ and intra-class correlation (ICC).

Results. Pseudo-planar PET EF estimates were concordant with planar ERNA (ccc $=0.81$, $P<.001)$ with a bias of $0 \%(95 \%$ CI $[-2 \% ; 3 \%]$, limits of agreement $[-11 \% ; 12 \%])$. Reproducibility was excellent and similar for both methods $(\mathrm{CoV}=2 \pm 1 \%$ and $3 \pm 2 \%$, $P=$ NS; ICC $=0.97$ and 0.92, for PET and ERNA, respectively). Measurements obtained in fully-tomographic mode were concordant with SPECT ERNA: ccc $=0.83$ and bias $=-3 \mathrm{~mL}$ for LV EDV, ccc $=0.92$ and bias $=0 \mathrm{~mL}$ for $\mathrm{LV} \mathrm{ESV}$, ccc $=0.89$ and bias $=-1 \%$ for $\mathrm{LV} \mathrm{EF}$ (all $P$ values $<.001$ for ccc, all biases not significant).

Conclusions. Gated first-pass ${ }^{18}$ F-FDG PET might stand as a relevant alternative to ERNA for $L V$ function assessment, enabling a joint evaluation of both therapeutic response and cardiac toxicity in oncologic patients receiving cardiotoxic chemotherapy. (J Nucl Cardiol 2019)

Key Words: ${ }^{18}$ F-FDG PET • first-pass • left ventricular function • left ventricular volume

Electronic supplementary material The online version of this article (https://doi.org/10.1007/s12350-019-01731-x) contains supplementary material, which is available to authorized users.

The authors of this article have provided a PowerPoint file, available for download at SpringerLink, which summarises the contents of the paper and is free for re-use at meetings and presentations. Search for the article DOI on SpringerLink.com.
Funding The authors have no funding source to declare. Reprint requests: Fayçal Ben Bouallégue, MD, PhD, Nuclear Medicine Department, Lapeyronie University Hospital, Avenue du Doyen Giraud 371, 34295 Montpellier Cedex 5, France. faycal.benbouallegue@umontpellier.fr 


\begin{tabular}{|ll|}
\hline \multicolumn{2}{|l|}{ Abbreviations } \\
LV & Left ventricle \\
RV & Right ventricle \\
EDV & End-diastole volume \\
ESV & End-systole volume \\
EF & Ejection fraction \\
ERNA & Equilibrium radionuclide angiography \\
SPECT & Single photon emission computerized \\
& tomography \\
\hline
\end{tabular}

\section{INTRODUCTION}

Left ventricle (LV) function assessment plays a critical role in baseline and follow-up assessment of patients with LV systolic ${ }^{1}$ or diastolic ${ }^{2}$ dysfunction, or receiving cardiotoxic chemotherapy. ${ }^{3}$ Standard LV function assessment based on the estimation of end diastole volume (EDV), end systole volume (ESV), and ejection fraction (EF) can be achieved using various non-invasive imaging techniques including echocardiography, cardiac magnetic resonance (CMR), and isotopic techniques. Gated equilibrium radionuclide angiography (ERNA), using either planar techniques or single photon emission computerized tomography (SPECT) and ${ }^{99 \mathrm{~m}} \mathrm{Tc}$-labeled red blood cells, has long proven to be a valuable and reproducible technique for cardiac function evaluation. ${ }^{4-7}$ Owing to its cost-effectiveness, simplicity, and high reproducibility, planar ERNA stands as the gold-standard for iterative LV EF assessment in adults. ${ }^{8}$ SPECT ERNA allows both LV volume and EF estimation and has been thoroughly validated against $\mathrm{CMR}^{5-7}$

First-pass radionuclide angiography using ${ }^{99 \mathrm{~m}} \mathrm{Tc}$ labeled agents has been extensively employed for LV and right ventricle (RV) function analysis. ${ }^{9}$ The steadily increasing use of PET in clinical routine reinforces the motivation to develop first-pass PET methods for cardiac function evaluation. ${ }^{10-12}$ Recent studies have highlighted the feasibility of LV function assessment using ${ }^{15} \mathrm{O}$-water PET and demonstrated the concordance of the produced estimates with those obtained using either CMR ${ }^{13,14}$ or myocardial perfusion SPECT. ${ }^{15}$ As a rule of thumb, the proposed methods are readily transposable to first-pass PET using any tracer, since the firstpass of the tracer through cardiac cavities provides -at least over a given time range- a set of vascular images that indirectly reflects cardiac function. Nowadays, the most employed PET tracer in clinical routine is ${ }^{18} \mathrm{~F}$ FDG, owing to its numerous indications in oncology and cardiology. The development of LV function assessment using first-pass ${ }^{18}$ F-FDG PET would substantially expand the diagnostic information provided by routine
${ }^{18}$ F-FDG PET explorations. By obviating the need for ERNA, it would also allow for higher patient comfort and lower received dose. Todica et al described LV volume and function measurements in healthy rats using gated first-pass ${ }^{18}$ F-FDG PET without significant difference compared to CMR in terms of ejection fraction. ${ }^{16}$ To our knowledge, no such measurements have been performed in humans to date.

TomPool is a free software developed in Montpellier University Hospital designed for semi-automated post-processing of equilibrium blood-pool SPECT images. It relies on a watershed immersion algorithm for ventricle segmentation and a deformable reference model for time-activity curve fitting. ${ }^{17-19}$ It has previously been validated against CMR for LV and RV function assessment. ${ }^{5}$ In a recent study, it was adapted to first-pass PET data post-processing and allowed to produce $\mathrm{LV}$ volume and function estimates using firstpass ${ }^{15} \mathrm{O}$-water PET that were in close agreement with those provided by gated myocardial perfusion SPECT. ${ }^{15}$

The present work aimed to investigate the feasibility of LV volume and function measurements using gated first-pass ${ }^{18}$ F-FDG PET, in both pseudo-planar and fully-tomographic post-processing settings, and to compare the produced volume and function estimates with those obtained using gated ERNA in both planar and SPECT modes.

\section{MATERIALS AND METHODS}

\section{Patients}

Twenty four patients aged $55 \pm 13$ years (range 2374 years, 13 men and 11 women) were prospectively recruited from the outpatients of the Nuclear Medicine Department at Montpelier University Hospital from December 2017 to November 2018. Included patients were referred for both ${ }^{18} \mathrm{~F}$-FDG PET and ERNA in routine condition. ${ }^{18} \mathrm{~F}$-FDG PET was performed for baseline or follow-up assessment of their cancer (18 malignant lymphomas, 4 breast cancer, 1 ovarian malignancy, and 1 cystic adenoid carcinoma). ERNA was performed for baseline or follow-up assessment of LV EF during cardiotoxic chemotherapy. The mean delay between ${ }^{18}$ F-FDG PET and ERNA was $6 \pm 6$ days (range 1-21 days). None of the recruited patients had documented cardiac disease. The study was approved by the Local Ethics Committee and all patients gave written informed consent.

\section{Gated Equilibrium Radionuclide Angiography Acquisition and Analysis}

Acquisition and post-processing parameters were set according to the international guidelines for ERNA. ${ }^{20,21}$ After in vivo labeling of red blood cells using $812 \pm 35$ (range 751913) $\mathrm{MBq}$ of ${ }^{99 \mathrm{~m}} \mathrm{Tc}$-pertechnetate, ERNA acquisitions were 
carried out using a dual-head Siemens Intevo camera equipped with low-energy high-resolution parallel-hole collimators. ECG gating used 16 time samples and a 10\% R-R interval acceptance window.

Planar acquisitions were performed over 7 minutes in the standard $35^{\circ}$ left-anterior-oblique direction. Planar images were sampled on a $64 \times 64$ matrix with a pixel size of $3.6 \mathrm{~mm}$, and post-processed using the manufacturer's dedicated software. The LV was automatically segmented using gradient-based delineation. Background noise subtraction was applied using a region of interest positioned bottom-left to the LV. Manual adjustment of the automated segmentation mask and background region position was performed whenever necessary.

SPECT acquisitions were performed using 32 projections per head and 20 second per projection. SPECT images were reconstructed using the manufacturer's specifications (3D OSEM, 4 subsets, 6 iterations, $9 \mathrm{~mm}$ Gaussian post-filtering), without attenuation and scatter correction. Images were sampled using $6.6 \mathrm{~mm}$ isotropic voxels. SPECT data were postprocessed using in-house software TomPool ${ }^{17-19}$ to produce estimates of LV EDV, ESV, and EF.

\section{Gated First Pass ${ }^{18}$ F-FDG PET Acquisition and Analysis}

Cardiac PET acquisitions were performed using a Siemens Biograph mCT Flow scanner in time-of-flight mode, following IV injection of $3.5 \mathrm{MBq} \cdot \mathrm{kg}^{-1}$ of ${ }^{18} \mathrm{~F}-\mathrm{FDG}(246 \pm 58 \mathrm{MBq}$, range 152-350 MBq). A transmission $\mathrm{CT}$ scan was obtained prior to PET for attenuation correction purpose (CT-AC). Emission list-mode data were acquired over 5 minutes starting from the IV injection of the tracer to the arm. After correction for random coincidences, scatter, and dead time, PET data were reconstructed twice. First, a dynamic sequence (30 frames of 10 seconds each) was obtained that allowed to identify the starting point for gated reconstructions. That starting point was set as the time at which a significant activity was seen inside the $\mathrm{LV}$. A second image reconstruction was then performed over the first-pass time range using CT-AC 3D OSEM (21 subsets, 3 iterations) with 8 -interval gating. The first-pass time range was either set to 1 or 3 minutes. Final image matrices were sampled on a $128 \times 128 \times 74$ grid with $3 \mathrm{~mm}$ cubic voxels. $3 \mathrm{D}$ Gaussian post-filtering was applied using an $8 \mathrm{~mm}$ kernel for pseudo-planar image extraction, and a $15 \mathrm{~mm}$ kernel for fullytomographic post-processing. A representative example of gated first-pass PET-CT images is shown in Figure 1. Gated first-pass blood-pool images were then post-processed using in-house software (TomPool) that was originally designed for ERNA and adapted for first-pass data analysis. A complete description of the algorithm can be found in. ${ }^{15,17-19}$

Regarding pseudo-planar image extraction, the workflow was as follows (see Figure 2). PET images were re-oriented into cardiac canonical axes using horizontal long axis then vertical long axis (VLA) views. VLA images were then summed along the left-right direction in order to compute a VLA phase image, which is a parametric map of the time of end-systole (pixel color codes the gating interval for which the pixel activity is the lowest). Since the volume changes of the ventricles occur in phase opposition compared to those of the atria, the valve plane (i.e., the separation between ventricular and atrial activity) could be easily located and manually positioned using the VLA phase image. Next, the activity located in front of the valve plane was summed along the antero-posterior direction to produce pseudo-planar images that reflect only ventricular activity. Last, automated segmentation of the LV on pseudo-planar images using thresholded region growing (with gradient-based LV/RV delineation) allowed to build an LV time-activity curve from which LV EF was inferred. Manual refinement of the produced contours was performed whenever necessary. Background noise subtraction was applied using a square region of interest of 5 pixels automatically positioned at a $24 \mathrm{~mm}$ distance left to the $\mathrm{LV}$. Thresholds ranging from $40 \%$ to $70 \%$ were tested in order to determine the optimal threshold value with respect to planar and SPECT ERNA.

Regarding fully-tomographic post-processing, a four-dimensional immersion algorithm was run that produced a partition of the gated images into $4 \mathrm{D}$ regions centered on local intensity maxima. Each region was then assigned either to the $\mathrm{LV}, \mathrm{RV}$, or extra-ventricular structures depending on the relative position of its barycentre with respect to the reference planes (septum and valve planes). The LV segmentation mask was obtained using a thresholding method at a fixed percentage of the 4D maximal intensity value inside the regions belonging to $\mathrm{LV}$. Thresholds ranging from $50 \%$ to $70 \%$ were tested in order to determine the optimal threshold value. LV volumes were computed using a count-based method in which the volume of each voxel was normalized by the ratio of its intensity to the LV maximal intensity. Ventricular ejection curves were fitted to the 8 time samples using a deformable curve model that allowed to estimate LV EDV, ESV, and EF. ${ }^{19}$

\section{Statistical Analysis}

Continuous variables are expressed as mean \pm standard deviation (min-max range). Parameters obtained using pseudoplanar PET were compared to those given by planar and SPECT ERNA. Parameters obtained using fully-tomographic PET were compared to those given by SPECT ERNA. Concordance and correlation between LV volume and function given by gated first-pass PET and those given by ERNA were assessed using Pearson's correlation coefficient (R), Lin's concordance correlation coefficient (ccc), and Bland-Altman analysis. Difference between homologous variables was characterized using a paired Student's t-test after checking data sample normalcy using the Kolmogorov-Smirnov test. Planar ERNA and pseudo-planar PET data were post-processed in a blinded fashion by 3 independent nuclear medicine specialists in order to assess the reproducibility of the LV EF measurements using the intra-class correlation (ICC) coefficient and the coefficient of variation $(\mathrm{CoV})$.

\section{RESULTS}

\section{Dosimetry}

Effective dose was $5.7 \pm 0.2$ (range 5.3-6.4) $\mathrm{mSv}$ for ERNA. Regarding PET-CT acquisition, total 

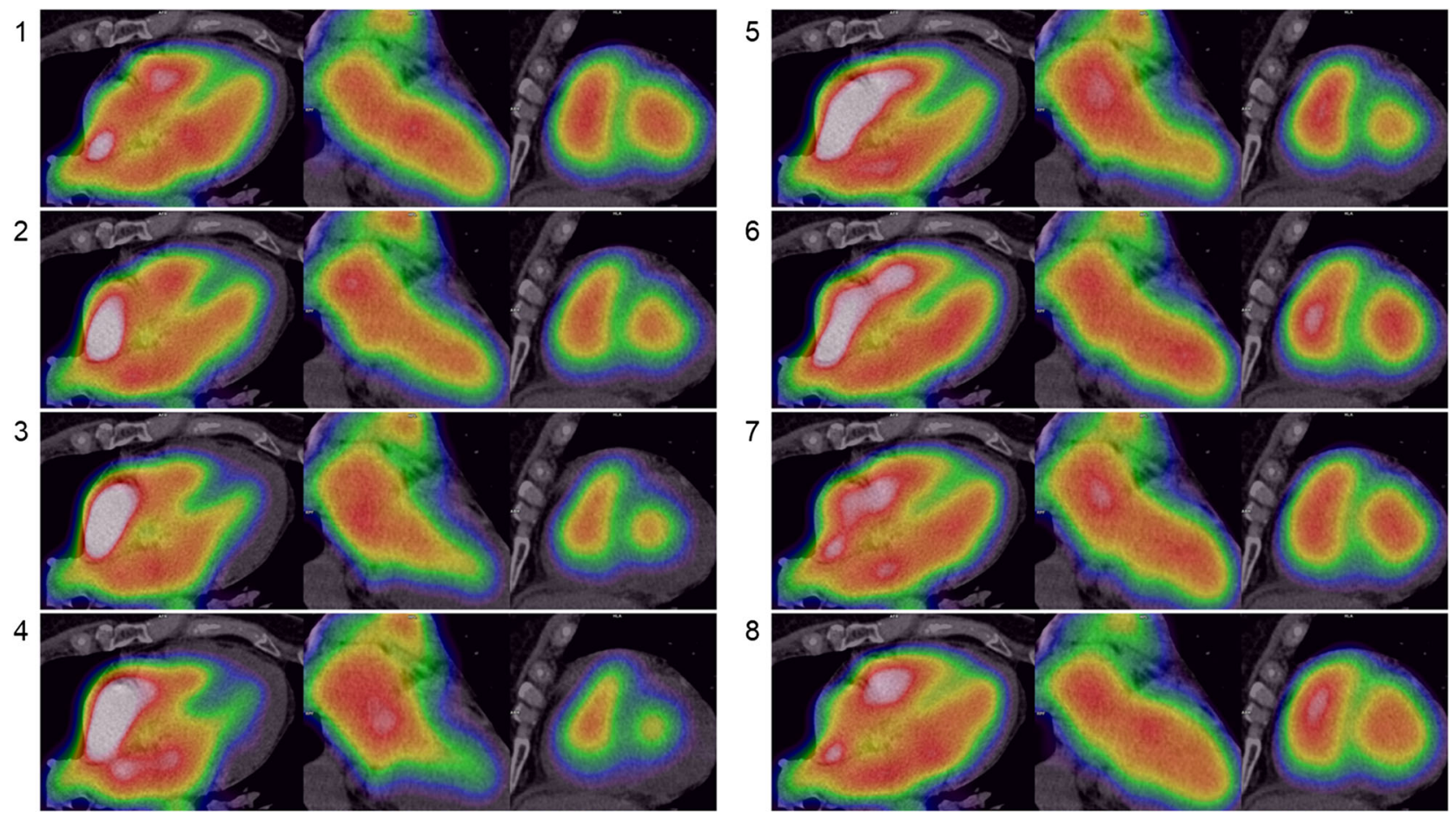

Figure 1. Representative gated first-pass ${ }^{18} \mathrm{~F}-\mathrm{FDG}$ PET-CT images. Images were reconstructed from gated list-mode data over 1 min during the first-pass of the tracer inside the LV (8-interval gating, $15 \mathrm{~mm}$ Gaussian post-filtering). Images are shown in horizontal long axis, vertical long axis, and short axis. Time frames are swept from top to bottom then left to right.

effective dose for the standard acquisition was $11.0 \pm 3.2$ (range 6.5-17.2) $\mathrm{mSv}$ (mean dose $4.7 \mathrm{mSv}$ for ${ }^{18} \mathrm{~F}-\mathrm{FDG}$ and $6.3 \mathrm{mSv}$ for chest-abdomen-pelvis CT). Additional effective dose due to cardiac CT was $3.8 \pm 1.0$ (range 1.7-5.4) $\mathrm{mSv}$.

\section{Pseudo-planar PET}

The optimal first-pass time range (either 1-minute or 3-minutes) was first assessed against planar ERNA as the reference. Visually, pseudo-planar images obtained using a first-pass time range of 1-minute had higher LVto-background contrast than 3-minutes images (see Online Figure 1) due to substantial myocardial and hepatic uptake on 3-minutes images. Quantitatively, 1minute LV EF estimates showed higher concordance with planar ERNA estimates than 3-minutes estimates (optimal Lin's concordance 0.82 and 0.63 respectively, see Online Table 1 and Online Figure 2 for details). Manual adjustment of the LV automatic segmentation was necessary in $2 / 24$ cases for planar ERNA, in 1/24 case for 1-minute pseudo-planar PET, and in 7/24 cases for 3-minutes pseudo-planar PET As such, 1-minute was considered as the optimal first-pass time range and all the results presented in the sequel are related to 1-minute PET images.

The best concordance and lowest bias of pseudoplanar PET with planar ERNA and SPECT ERNA was achieved using 50\% and 65\% segmentation thresholds respectively. Figure 3 shows the scatter plots and BlandAltman diagrams assessing the concordance between LV EF obtained using pseudo-planar PET and planar (top) and SPECT (bottom) ERNA. With respect to planar ERNA, using a 50\% threshold, Lin's concordance was $0.81(P<.001)$ and bias was $0 \%$ (not significant, $95 \%$ CI $[-2 \% ; 3 \%]$, limits of agreement [-11\%; $12 \%]$ ). As demonstrated by the Bland-Altman diagram, there was a moderate albeit significant trend towards an underestimation of low EFs and overestimation of high EFs using pseudo-planar PET. With respect to SPECT ERNA, using a $65 \%$ threshold, Pearson's correlation and Lin's concordance were both $0.75(P<.001)$ and bias was $0 \%$ (not significant, 95\% CI [-4\%; 4\%], limits of agreement [-17\%; 18\%]).

Planar ERNA LV EF reproducibility was characterized by a $\mathrm{CoV}$ of $3 \pm 2 \%$ (range $[0 ; 10 \%]$ ) and an ICC coefficient of 0.92 . Pseudo-planar PET $(50 \%$ threshold) LV EF reproducibility was characterized by 


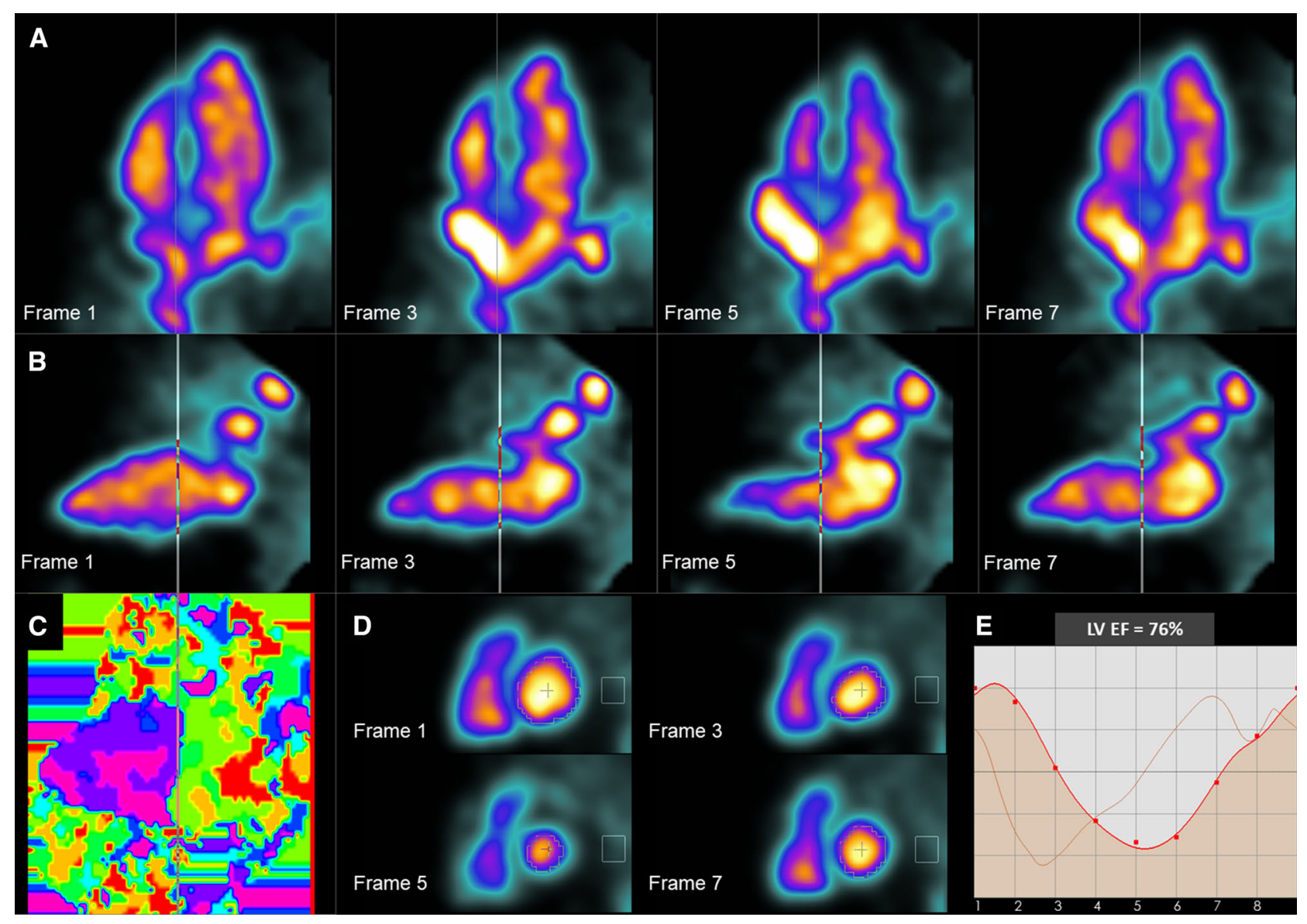

Figure 2. Workflow for LV EF estimation using pseudo-planar PET images. PET images are reoriented into canonical axes using horizontal long axis (A) then vertical long axis (B) views. VLA images are then summed along the left-right direction in order to compute a VLA phase image (C) that allows to manually position the valve plane (highlighted vertical line). Next, the activity located in front of the valve plane is summed along the anterior-posterior direction to produce pseudo-planar images of ventricular activity (D). Last, automated segmentation of the LV on pseudo-planar images using thresholded region growing and gradient-based delineation allows to build an LV time-activity curve (E).

a $\mathrm{CoV}$ of $2 \pm 1 \%$ (range $[0 ; 6 \%]$ ) and an ICC coefficient of 0.97 . No statistical difference was found between the $\mathrm{CoV}$ obtained in planar ERNA and those obtained in pseudo-planar PET.

\section{Fully-tomographic Post-processing}

LV delineation using a $60 \%$ segmentation threshold yielded the highest concordance and lowest bias of firstpass PET with SPECT ERNA in terms of LV EDV, ESV, and EF (see Online Table 2 for details). Figure 4 shows the scatter plots and Bland-Altman diagrams assessing the concordance between LV EDV, ESV, and EF (from left to right) obtained using fully-tomographic PET and SPECT ERNA. Lin's concordance was 0.83 for
LV EDV, 0.92 for LV ESV, and 0.89 for LV EF (all $P$ values $<.001$ ). Bias was $-3 \mathrm{~mL}$ (not significant, $95 \%$ CI $[-7 ; 1] \mathrm{mL}$, limits of agreement $[-20 ; 14] \mathrm{mL}$ ) for LV EDV, $0 \mathrm{~mL}$ (not significant, $95 \% \mathrm{CI}[-2 ; 2] \mathrm{mL}$, limits of agreement $[-9 ; 9] \mathrm{mL}$ ) for LV ESV, and $-1 \%$ (not significant, 95\% CI [-4\%; 1\%], limits of agreement $[-12 \% ; 10 \%])$ for LV EF.

Figure 5 shows a representative example of SPECT ERNA (left) and first-pass PET (right). From top to bottom are displayed the 3D segmentations (raw volumes and parametric surface rendering) at end diastole and end systole, and the LV time-activity curves. Cine gated images are provided as supplemental material (see Online Figure 3). 
LV EF (\%)
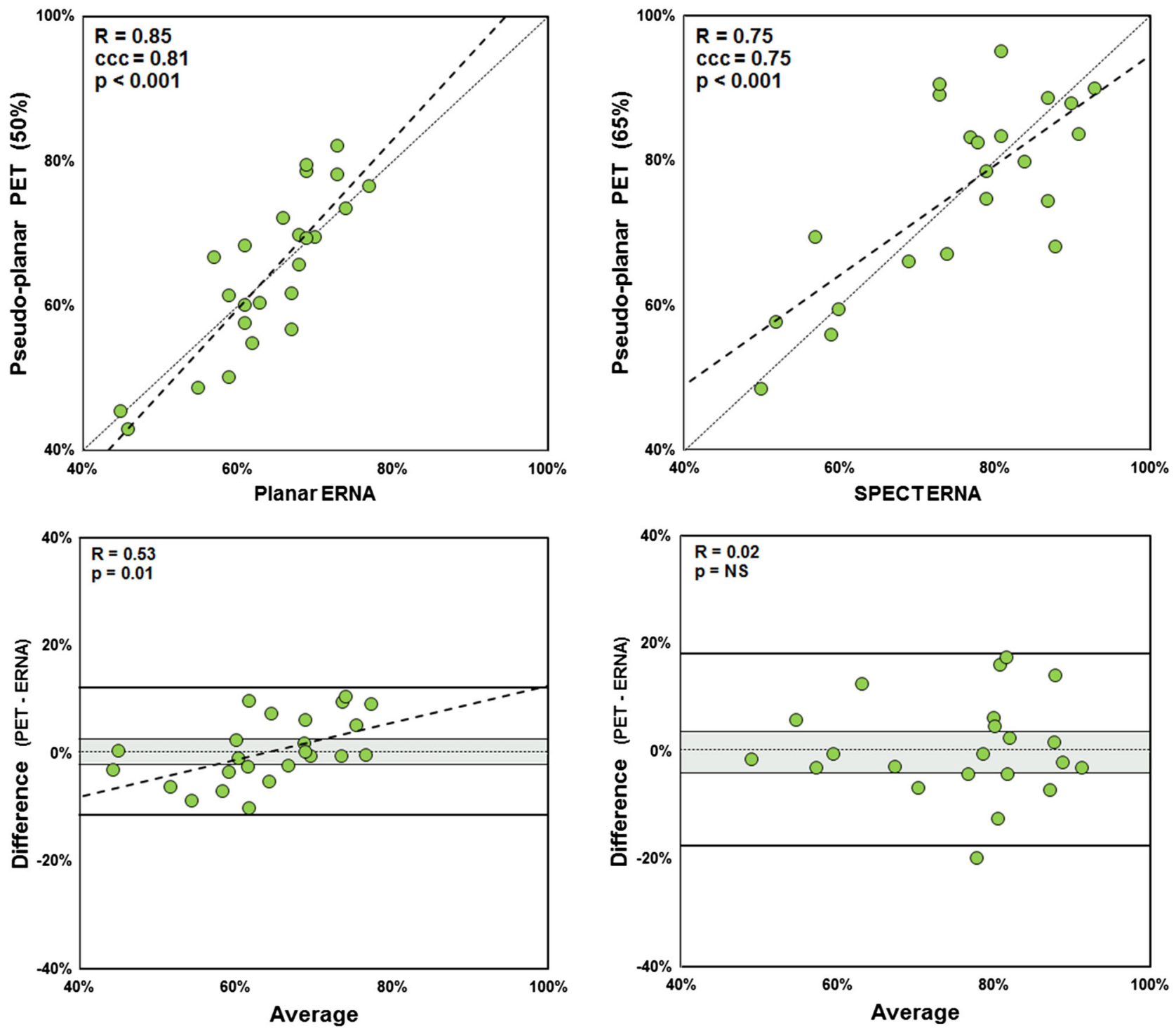

Figure 3. Comparison between LV EF estimates obtained using pseudo-planar gated first-pass PET images and (left) planar and (right) SPECT ERNA. Top: scatter plots. The dotted lines indicate the perfect identity. The dashed lines stand for the linear regression. Bottom: Bland-Altman diagrams. The dotted lines indicate the mean difference (grayed is the $95 \%$ confidence interval) and the plain lines the $95 \%$ limits of agreement. The dashed line stands for the linear regression (not shown if not significant). $R$ Pearson's correlation; $c c c$ Lin's concordance correlation coefficient.

\section{DISCUSSION}

In the present study, we assessed the feasibility and relevance of LV EF and volume measurements from gated first-pass ${ }^{18}$ F-FDG PET. First-pass PET images were post-processed in both pseudo-planar and fullytomographic modes. The motivation to develop a pseudo-planar approach was based on the fact that, owing to its high reproducibility, planar ERNA stands as the gold-standard isotopic method for iterative LV EF evaluation and detection of drug-induced cardiac toxicity. ${ }^{21,22}$ Besides, pseudo-planar post-processing is much more practical in terms of post-processing time (less than one minute compared to several minutes for the fully-tomographic approach), making it readily implementable in clinical routine. As such, pseudoplanar techniques based on re-projection of SPECT data have already been developed using standard and dedicated cameras. ${ }^{23-25}$ 
To our knowledge, this is the first proof of concept for $\mathrm{LV}$ function assessment using first-pass ${ }^{18} \mathrm{~F}-\mathrm{FDG}$ PET in humans along with a post-processing workflow compatible with patient throughput in nuclear medicine labs. In this context, the use of the routine tracer ${ }^{18} \mathrm{~F}$ FDG appears crucial since previous attempts to asses LV function using first-pass PET methods relied on ${ }^{15} \mathrm{O}$ water or ${ }^{15} \mathrm{O}$-labeled carbon monoxide which are restricted to institutions equipped with an on-site cyclotron. ${ }^{13-15,25}$ In a patient viewpoint, there are mainly two expected benefits from ${ }^{18}$ F-FDG PET-based LV function assessment. First, it allows to evaluate both disease extension (or therapeutic response) and LV function in a single examination. Second, it enables a moderate albeit significant reduction in terms of effective dose $(3.8 \mathrm{mSv}$ for cardiac CT vs $5.7 \mathrm{mSv}$ for ERNA). At our institution, a large proportion of patients addressed for concomitant ${ }^{18}$ F-FDG PET and ERNA are affected by hematologic malignancies. In these often young patients for whom a long follow-up including repeated isotopic explorations is required, dosimetric considerations are of critical importance. ${ }^{26}$

Dynamic PET acquisitions were performed over 5 minutes in order to estimate the optimal time range for first-pass image extraction. Due to substantial myocardial uptake (with respect to vascular activity) from the first minutes following tracer injection, ${ }^{27}$ the PET images with the higher LV-to-background contrast were those obtained using a short first-pass time range of 1 minute. Although the kinetics of ${ }^{18} \mathrm{~F}$-FDG myocardial uptake is known to be highly variable and dependent upon fasting state and plasma glucose and insulin levels, ${ }^{28}$ myocardial uptake was negligible on 1-minute images in all of our patients. Owing to that high contrast, the absence of splenic uptake interfering with background correction, and the cancelation of atrial activity on the short-axis images obtained by re-projection, the LV EF estimates produced by the pseudo-planar method
$\operatorname{LV} \operatorname{EDV}(\mathrm{mL})$
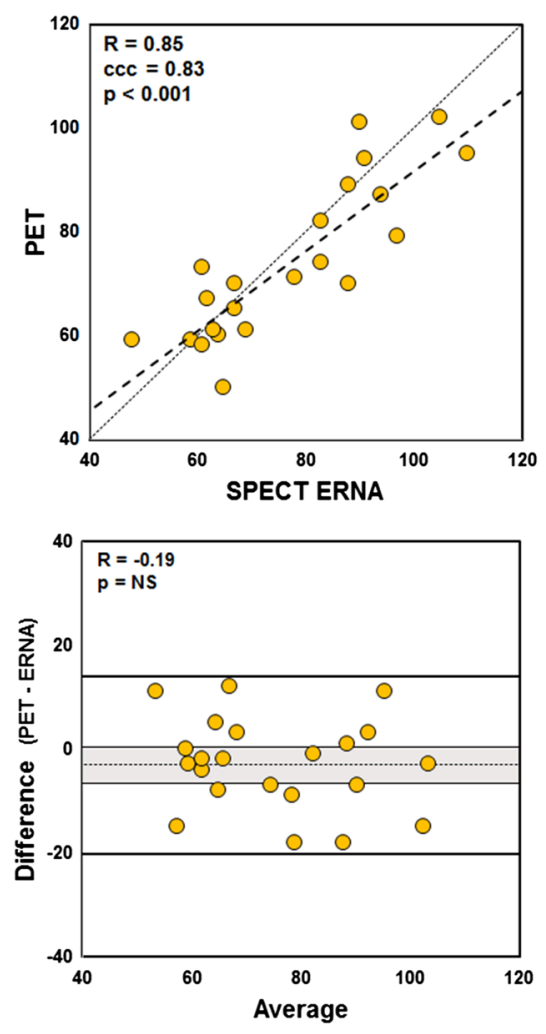

$\operatorname{LV} \operatorname{ESV}(\mathrm{mL})$
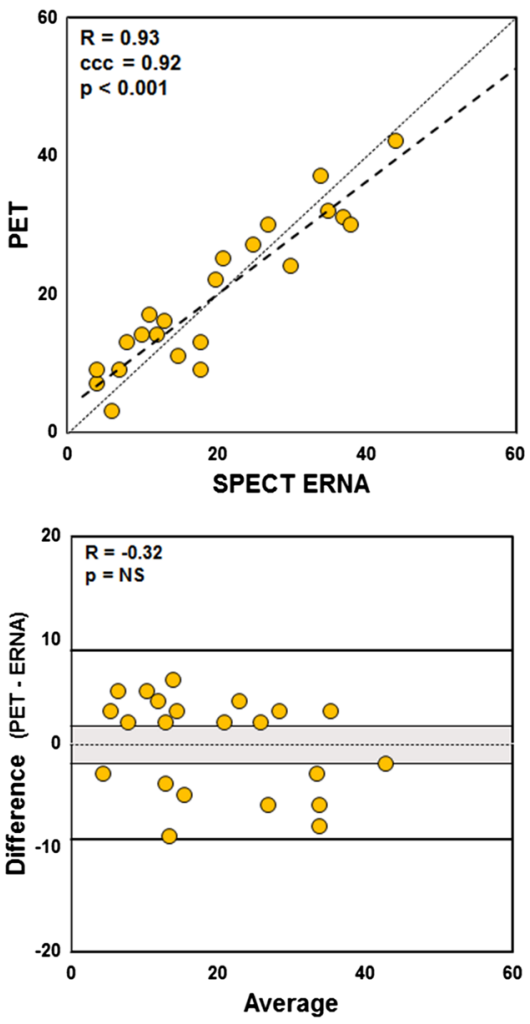

LV EF (\%)
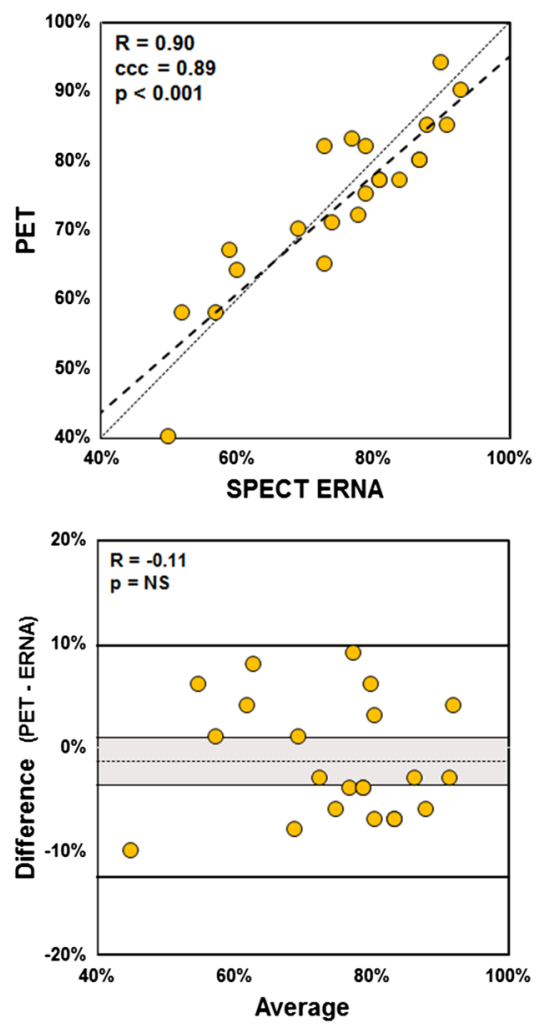

Figure 4. Comparison between LV EDV, ESV, and EF estimations obtained using gated first-pass PET and SPECT ERNA. Top: scatter plots. The dotted lines indicate the perfect identity. The dashed lines stand for the linear regression. Bottom: Bland-Altman diagrams. The dotted lines indicate the mean difference (grayed is the $95 \%$ confidence interval) and the plain lines the $95 \%$ limits of agreement. $R$ Pearson's correlation, $c c c$ Lin's concordance correlation coefficient. 


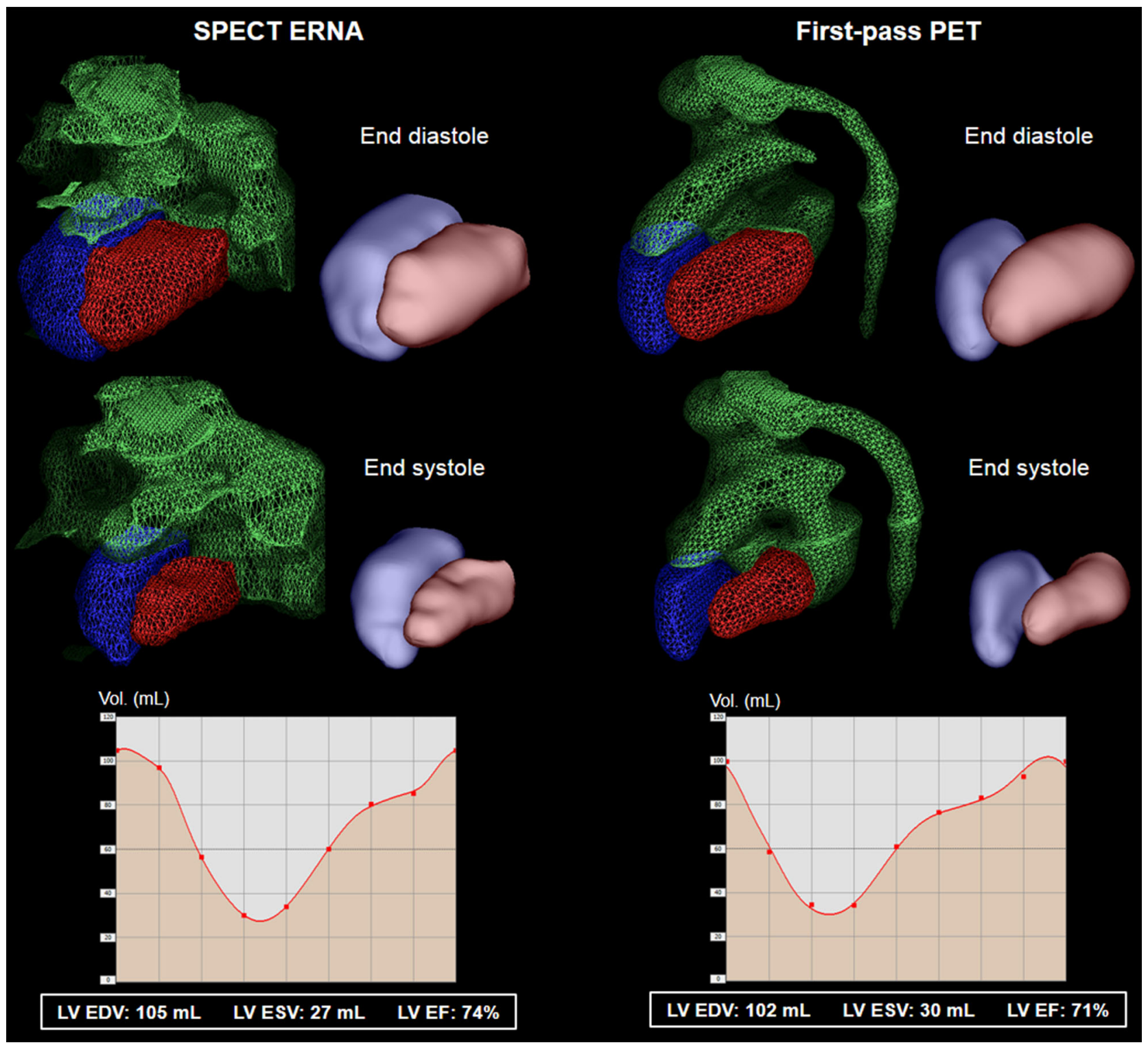

Figure 5. Representative example of SPECT ERNA (left) and first-pass PET (right) in patient \#11 (male, 72 years, baseline assessment in a follicular lymphoma). From top to bottom are shown the 3D segmentations (raw volumes and parametric surface rendering: left ventricle in red, right ventricle in blue, extra-ventricular structures in green) at end diastole and end systole, and the LV time-activity curves.

showed favorable inter-operator reproducibility (mean $\mathrm{CoV} 2 \%)$. The variability of planar ERNA LV EF measurements was slightly higher (mean $\mathrm{CoV} 3 \%$ ) and similar to that reported in the literature. ${ }^{29,30}$ Using an adapted segmentation threshold at $50 \%$ of the global maximal LV activity on pseudo-planar images, there was an excellent agreement between the two methods (no significant bias, limits of agreement around $\pm 10 \%$ ). Importantly, considering the reference cutoff at $50 \%$ to detect LV dysfunction in patient receiving cardiotoxic therapies, ${ }^{8}$ all patients were identically classified using planar ERNA and pseudo-planar PET (22 normal and 2 altered LV EF). It has however to be noted that a trend towards a proportional bias is observed on the BlandAltman diagram. The mean bias was not significant because the optimal segmentation threshold was set so as to maximize the concordance between planar ERNA and pseudo-planar PET EF estimates. The proportional bias may be explained by the fact that pseudo-planar PET post-processing allows to exclude atrial activity by 
re-projecting only ventricular activity. Using planar ERNA, atrial activity may overlap ventricular activity, leading to relative EF under-estimation especially at high $\mathrm{EF}$ values.

Concordance of pseudo-planar LV EF estimates with those provided by SPECT ERNA was moderate, especially for high EF values (limits of agreement around $\pm 20 \%$ ). Nevertheless, Pearson's correlation $(\mathrm{R}=0.75)$ was similar to that reported in the literature between planar ERNA and SPECT ERNA processed using standard commercial software. ${ }^{31}$

Although 16-interval gating is recommended for planar ERNA and sometimes preferred for SPECT ERNA,${ }^{24,25}$ it has been shown that LV EF estimates obtained using 8-frame gated SPECT ERNA do not significantly differ from those obtained using 16 -frame gating ${ }^{32}$ and are concordant with CMR measurements. ${ }^{5}$ In our study, gated PET data were sampled using 8 frames/cycle. This was primarily motivated by the inherent low-count nature of short duration first-pass data, and in line with previous studies exploring the feasibility of first-pass PET LV EF assessment. ${ }^{13-16}$

The fully-tomographic post-processing of first-pass PET data provided LV assessment that was highly concordant with SPECT ERNA measurements in terms of LV EDV, ESV, and EF. Volume measurements relied on a count-based approach assuming a uniform activity distribution inside the LV. This method is supposed to account for signal blurring at the ventricle edges due to partial volume effect and motion artifacts. When passing from equilibrium to first-pass imaging, the uniformity assumption was reasonably maintained for what concerns the LV since tracer dilution in blood flow was sufficient downstream the pulmonary circulation. ${ }^{33}$ The optimal threshold value for LV segmentation was found to be $60 \%$, which is higher than the optimal threshold previously reported using first-pass ${ }^{15} \mathrm{O}$-water PET. ${ }^{15}$ The discrepancy between the two threshold values was likely due to a better resolution and contrast in ${ }^{18} \mathrm{~F}$-FDG images compared to ${ }^{15} \mathrm{O}$-water images (smaller positron range, slower tracer diffusion to the myocardium) and to the different gold-standards employed (ERNA vs myocardial perfusion SPECT). Although feasible using first-pass PET methods, ${ }^{15}$ right ventricle (RV) analysis was not performed because it was beyond the scope of the present study and because gold-standard assessment of RV function was not available in our cohort.

Last, the technique presented in the present study should be feasible and relevant in the frame of cardiac PET for which a hypoglycemic regime and/or a euglycemic hyperinsulinemic clamp is usually performed. Indeed, in either case myocardial uptake is expected to be negligible during the first minutes post-injection that are exploited for first-pass blood-pool analysis. ${ }^{34}$

\section{NEW KNOWLEDGE GAINED}

This study demonstrates that LV function assessment is feasible using gated first-pass ${ }^{18} \mathrm{~F}$-FDG PET-CT, yielding higher patient comfort and lower total dosimetry. First-pass PET images are mineable using either pseudo-planar or fully-tomographic approaches. The latter provides a complete characterization of $\mathrm{LV}$ functional parameters (including volumes and $\mathrm{EF}$ ) that is in good agreement with SPECT ERNA assessment. The pseudo-planar workflow yields reproducible LV EF estimates that are highly concordant with planar ERNA measurements. Its implementation should easily fit within a clinical routine framework.

\section{CONCLUSION}

Gated first-pass ${ }^{18}$ F-FDG PET might stand as a relevant alternative to ERNA for LV function assessment, enabling a joint evaluation of both therapeutic response and cardiac toxicity in oncologic patients receiving cardiotoxic chemotherapy.

\section{Disclosure}

The authors have no conflict of interest to disclose.

\section{References}

1. Wang TJ, Evans JC, Benjamin EJ, Levy D, LeRoy EC, Vasan RS. Natural history of asymptomatic left ventricular systolic dysfunction in the community. Circulation 2003;108:977-82.

2. Flachskampf FA, Biering-Sørensen T, Solomon SD, Duvernoy O, Bjerner T, Smiseth OA. Cardiac imaging to evaluate left ventricular diastolic function. JACC Cardiovasc Imaging 2015;8:1071-93.

3. Murbraech K, Smeland KB, Holte H, Loge JH, Lund MB, Wethal $\mathrm{T}$, et al. Heart failure and asymptomatic left ventricular systolic dysfunction in lymphoma survivors treated with autologous stemcell transplantation: A national cross-sectional study. J Clin Oncol 2015;33:2683-91.

4. van Royen N, Jaffe CC, Krumholz HM, Johnson KM, Lynch PJ, Natale D, et al. Comparison and reproducibility of visual echocardiographic and quantitative radionuclide left ventricular ejection fractions. Am J Cardiol 1996;77:843-50.

5. Sibille L, Ben Bouallègue F, Bourdon A, Micheau A, VernhetKovacsik H, Mariano-Goulart D. Comparative values of gated blood-pool SPECT and CMR for ejection fraction and volume estimation. Nucl Med Commun 2011;32:121-8.

6. Nichols KJ, Van Tosh A, Wang Y, Palestro CJ, Reichek N. Validation of gated blood-pool SPECT regional left ventricular function measurements. J Nucl Med 2009;50:53-60.

7. Ioannidis JP, Trikalinos TA, Danias PG. Electrocardiogram-gated single-photon emission computed tomography versus cardiac magnetic resonance imaging for the assessment of left ventricular volumes and ejection fraction: A meta-analysis. J Am Coll Cardiol 2002;39:2059-68. 
8. Jiji RS, Kramer CM, Salerno M. Non-invasive imaging and monitoring cardiotoxicity of cancer therapeutic drugs. J Nucl Cardiol 2012;19:377-88.

9. Friedman JD, Berman DS, Borges-Neto S, Hayes SW, Johnson LL, Nichols KJ, et al. First-pass radionuclide angiography. J Nucl Cardiol 2006;13:e42-55.

10. Chen EQ, MacIntyre WJ, Fouad FM, Brunken RC, Go RT, Wong $\mathrm{CO}$, et al. Measurement of cardiac output with first-pass determination during rubidium-82 PET myocardial perfusion imaging. Eur J Nucl Med 1996;23:993-6.

11. Kreissl MC, Wu HM, Stout DB, Ladno W, Schindler TH, Zhang $\mathrm{X}$, et al. Noninvasive measurement of cardiovascular function in mice with high-temporal-resolution small-animal PET. J Nucl Med 2006;47:974-80.

12. Knaapen P, Lubberink M, Rijzewijk LJ, van der Meer RW, Unger $\mathrm{M}$, Germans T, et al. Stroke volume measurements with first-pass dynamic positron emission tomography: Comparison with cardiovascular magnetic resonance. J Nucl Cardiol 2008;15:218-24.

13. Driessen RS, van Timmeren JE, Stuijfzand WJ, Rijnierse MT, Danad I, Raijmakers PG, et al. Measurement of LV volumes and function using oxygen-15 water-gated PET and comparison with CMR imaging. JACC Cardiovasc Imaging 2016;9:1472-4.

14. Nordström J, Kero T, Harms HJ, Widström C, Flachskampf FA, Sörensen J, et al. Calculation of left ventricular volumes and ejection fraction from dynamic cardiac-gated 15O-water PET/CT: 5D-PET. EJNMMI Phys 2017;4:26.

15. Ben Bouallègue F, Mariano-Goulart D, Agostini D, Manrique A. Feasibility of biventricular volume and function assessment using first-pass gated ${ }^{15} \mathrm{O}$-water PET. EJNMMI Res 2018;8:92.

16. Todica A, Böning G, Lehner S, Weidl E, Cumming P, Wängler C, et al. Positron emission tomography in the assessment of left ventricular function in healthy rats: A comparison of four imaging methods. J Nucl Cardiol 2013;20:262-74.

17. Mariano-Goulart D, Collet H, Kotzki PO, Zanca M, Rossi M. Semi-automatic segmentation of gated blood-pool emission tomographic images by watersheds. Application to the determination of right and left ejection fractions. Eur J Nucl Med 1998;25:1300-7.

18. Mariano-Goulart D, Piot C, Boudousq V, Raczka F, Comte F, Eberlé $\mathrm{MC}$, et al. Routine measurements of left and right ventricular output by gated blood pool emission tomography in comparison with thermodilution measurements: A preliminary study. Eur J Nucl Med 2001;28:506-13.

19. Caderas de Kerleau C, Ahronowitz E, Rossi M, Mariano-Goulart D. Automatic generation of noise-free time-activity curve with gated blood-pool emission tomography using deformation of a reference curve. IEEE Trans Med Imaging 2004;23:485-91.

20. Corbett JR, Akinboboye OO, Bacharach SL, Borer JS, Botvinick $\mathrm{EH}$, DePuey EG, et al. Equilibrium radionuclide angiocardiography. J Nucl Cardiol 2006;13:e56-79.

21. Hesse B, Lindhardt TB, Acampa W, Anagnostopoulos C, Ballinger $\mathrm{J}$, Bax JJ, et al. EANM/ESC guidelines for radionuclide imaging of cardiac function. Eur $\mathbf{J}$ Nucl Med Mol Imaging 2008;35:851-85
22. Ganz WI, Sridhar KS, Ganz SS, Gonzalez R, Chakko S, Serafini A. Review of tests for monitoring doxorubicin-induced cardiomyopathy. Oncology 1996;53:461-70.

23. Bartlett ML, Srinivasan G, Barker WC, Kitsiou AN, Dilsizian V, Bacharach SL. Left ventricular ejection fraction: Comparison of results from planar and SPECT gated blood-pool studies. J Nucl Med 1996;37:1795-9.

24. Wells RG, Marvin B, Kovalski G, Ruddy TD. Planar radionuclide angiography with a dedicated cardiac SPECT camera. J Nucl Cardiol 2013;20:358-66.

25. Hofman HA, Knaapen P, Boellaard R, Bondarenko O, Götte MJ, van Dockum WG, et al. Measurement of left ventricular volumes and function with O-15-labeled carbon monoxide gated positron emission tomography: Comparison with magnetic resonance imaging. J Nucl Cardiol 2005;12:639-44.

26. Nievelstein RA, Quarles van Ufford HM, Kwee TC, Bierings MB, Ludwig I, Beek FJ, et al. Radiation exposure and mortality risk from CT and PET imaging of patients with malignant lymphoma. Eur Radiol 2012;22:1946-54.

27. Bøtker HE, Goodwin GW, Holden JE, Doenst T, Gjedde A, Taegtmeyer H. Myocardial glucose uptake measured with fluorodeoxyglucose: A proposed method to account for variable lumped constants. J Nucl Med 1999;40:1186-96.

28. Choi Y, Brunken RC, Hawkins RA, Huang SC, Buxton DB, Hoh CK, et al. Factors affecting myocardial 2-[F-18]fluoro-2-deoxy-Dglucose uptake in positron emission tomography studies of normal humans. Eur J Nucl Med 1993;20:308-18.

29. Okada RD, Kirshenbaum HD, Kushner FG, Strauss HW, Dinsmore RE, Newell JB, et al. Observer variance in the qualitative evaluation of left ventricular wall motion and the quantitation of left ventricular ejection fraction using rest and exercise multigated blood pool imaging. Circulation 1980;61:128-36.

30. Heidendal GA, Bezemer PD, Koopman PA, den Hollander W, Teule GJ, van der Wall EE, et al. Reproducibility of ejection fraction measurements by gated equilibrium blood pool scintigraphy. Eur J Nucl Med 1983;8:467-70.

31. Harel F, Finnerty V, Ngo Q, Grégoire J, Khairy P, Thibault B. SPECT versus planar gated blood pool imaging for left ventricular evaluation. J Nucl Cardiol 2007;14:544-9.

32. Kim SJ, Kim IJ, Kim YS, Kim YK. Gated blood pool SPECT for measurement of left ventricular volumes and left ventricular ejection fraction: Comparison of 8 and 16 frame gated blood pool SPECT. Int J Cardiovasc Imaging 2005;21:261-6.

33. Dokoumetzidis A, Macheras P. A model for transport and dispersion in the circulatory system based on the vascular fractal tree. Ann Biomed Eng 2003;31:284-93.

34. Knuuti MJ, Nuutila P, Ruotsalainen U, Saraste M, Härkönen R, Ahonen A, et al. Euglycemic hyperinsulinemic clamp and oral glucose load in stimulating myocardial glucose utilization during positron emission tomography. J Nucl Med 1992;33:1255-62.

Publisher's Note Springer Nature remains neutral with regard to jurisdictional claims in published maps and institutional affiliations.' 